\title{
A Study on Sports Team of Colleges Physical Quality Training
}

\author{
Zhongfeng $\operatorname{Sun}^{1}$ \\ ${ }^{1}$ Department of Physical Education, Qingdao University of Science \& Technology, Laoshan District, Qingdao, \\ Shandong, China \\ Correspondence: Zhongfeng Sun, Department of Physical Education, Qingdao University of Science \& \\ Technology, Laoshan District, Qingdao 266061, Shandong, China. E-mail: sunzhongfeng1976@163.com
}

Received: April 19, 2015 Accepted: April 28, 2015 Online Published: April 30, 2015

doi:10.5539/ass.v11n13p28 URL: http://dx.doi.org/10.5539/ass.v11n13p28

\begin{abstract}
Physical training is an important and indispensable part in sports training. School physical education is also carried out around the physical training. Therefore, physical education teachers and coaches should attach great importance to physical training.
\end{abstract}

Keywords: colleges and universities, quality, training method

According to the manual classification, "physical strength" can be divided into "action strength" and "defense strength" two kinds. Action strength is referred to the ability that be demonstrated by run, jump and playing and it is essential to the body during exercise and physical activity, also, these strengths have some kind of relationships with the size. However, Defense strength refers to the ability that how extent the body can endure the cold, heat...etc..., and the adaptability to these situations. Such as, whether to be get colds easily, whether to be appeared the cerebral anemia phenomenon when stand for a long time, and all these problems are belong to the defense strength problem. So, to engage in sports activities, the action strength is essential, and in order to improve the action strength the physical training is necessary.

Action strength including physical strength (musical strength), endurance, speed, dexterity and flexibility, in which the strength, endurance and speed is the most important three qualities.

The strength, speed and endurance can be demonstrated as a shaft, and the length of the shaft is the quality of body. If the body quality is better the shaft is longer, otherwise, the length of shaft is shorter. So, there will be a plane between the two axes, and an overall volume of the physical quality will be formed among these three axes, so it can easily reveal the differences in the level of physical training of athletes.

Between the power and speed of the two axes is the explosive (speed power) plane, between the strength and endurance is the strength endurance plane, And between the speed and endurance of the two axes is speed and endurance plane.

If the strength and speed quality are strong but the endurance is poor for an athlete, the endurance of the explosive force is also not good for him. So, the volume of the whole action strength is very small, and the majority of sprinters belong to this type. If the endurance is strong but the strength and speed are poor for an athlete, the whole action physical volume is also very small, and most of the marathon runners are of this type. It can be seen that simply in developing the training of speed, strength or endurance is not the best way. The athlete should training these three qualities at the same time, which is the best way to enhance physical strength, in other words, it can expand the whole volume of it.

\section{Principles of Physical Training}

Physical training to be able to have a good effect on athlete's heart, circulatory and respiratory system, but also can make the muscles and nervous system in a good development. In order to illustrate the effect of physical training on the body, we can sum up following contents:

Starting a physical training, we should understand what effects that we want to get in first and then decide to choose different training methods, according to this objective. Because only the athletes achieve the enough training can grasp the sports skills and exercise effects, also, the selection objectives and training methods that they chose should be compliance with their tasks, so they must clearly the objective of training.

Because of people's gender, age, physical fitness, health and hobbies are different; the physical training must 
consider these differences. For example, because of the age difference, the intensity should not be too heavy for the teenagers at 16-18 years old. At the same time, because of the differences of personal physical, we should accord to the different conditions of muscle type to choose the different training methods, such as to the muscular, obesity, thin physics, we should select and carry on different training method.

Following principles should be obeyed in the physical training:

1) The Overload principle: It must be a certain overload in training, and after a certain period of training, the original overload training frequency should be enhanced increasingly, that is to say, after that the overload level must be more than the original load level.

2) The Progressive principle: Gradually increase the amount of exercise. The effects of the physical training are not good when the amount of exercise is too small or too large in the training, which also may cause the negative effect. For example, when taking the heavy barbell exercises, the heavier barbell may easy to cause an accident or muscle strain. Therefore, everyone should gradually increase the amount of exercise in the training according to the condition of their physical strength.

3) The continuity principle: if not persist very well, Physical training is not easy to receive the aimed effects. So, the physical training should be maintained in a constant practice state, through constant stimulation, physical training the body every day or every other day is a best way. For the physical training, if you relax the body for a long time, the physical strength may be fade gradually.

4) The individual principle: Everyone should not do the same content of physical training, which request the players adopt different training methods in training from man to man, and players also arrange different physical exercise amount according to their physical conditions, so, in the training, it should make the amount of exercise and exercise intensity are not as the same as all the team members.

5) The comprehensive principle: If the body gets an all-round development, after a basis of comprehensive development for the body, the player can lay particular emphasis turn to the special quality development.

Physical training can improve the certain quality especially according to the different physical training task, such as taking strength training to improve the muscle strength. The long-distance running training can increase the durability of response, and agility training can improve the speed, also, when taking physical training, the player can also carry out comprehensive physical training.

\section{Strength Training}

Strength training can make the muscle fibers and muscle mass bigger, which is to say this training can make the whole muscle cross section increases bigger, so, those kind of person who have not muscular strength can make up this flaw with this training method. The changes of muscle fiber before and after training are shown as follows:

a) Muscular strength. It can be divided a short moment of maximum force and muscular endurance strength from the force of the play to distinguish. From muscle contraction to distinguish, it can be divided static and dynamic force; from the initiative to distinguish, it can be divided into active muscle strength and passive muscle power. Muscle strength and muscle thickness is directly proportional. In order to effectively improve the muscle strength, the overload method is introduced as follows.

1) The maximum muscle strength: Taking in a possible short time to get to the individual maximum overload training method.

2) Muscle endurance: Taking closely to a personal $1 / 3$ maximum overload training to repeated practice method.

3) Static strength: Taking closely to a personal maximum overload training to practice method.

4) Dynamic strength (explosive strength): Taking closely to a personal $1 / 3-2 / 3$ maximum overload and do training as fast as possible method.

b) Strength training. The strength of people including the local strength (including arm strength, leg strength, grip strength, back strength and abdominal muscle strength) and general strength. Therefore, the people should target to the development of local and systemic exercise muscle strength in the strength training.

To improve the strength, it must be the heavy loads, such as negative barbells, dumbbells, Kettle bell, using a pulley fixed expander and so on, or on its own weight or others for resistance. It not only can improve the muscle activity, it also can be the weight-bearing exercises as auxiliary sports activities, most of all, It can also play a role in health care in correcting the body posture. The overload training should follow the principle of gradual, take closely to a personal 2/3 maximum overload training, and repeated practice method, which would improve 
the biggest muscle strength. When aimed to enhance muscle endurance, it should adopt the $1 / 3-2 / 3$ individual maximum overload training, also, it can increase the number of repeats, which should accord to the aimed development muscles to practice, and then combine all these of training practices, which would be better for the training effect.

The training effect and disappearing reflects the relationship between training time interval and the length of the training period with increase the rate of muscle strength:

1) Strength training of a long time interval is not effective, strength training within two days of not less than one time will get a best effect, however, strength training within a five day, the effect is equivalent to $50 \%$ of two days of not less than one time training.

2) If Strength training is day by day, the effective is very fast in the short term, but after the 1.5 times of the training period, it will drop to the original level. After 24 weeks of training, the strength is growing fast, but stopped after 32 weeks of training, and down to the original level, and the training effect disappeared time equivalent to 1.5 times of training effect time. It shows that soon gained strength also disappear fast. On the contrary, it has gradually gained strength after training; it will take a short long time after stopping training. Strength training for a long time at 2-3 times for a week, this would be useful to maintain its training effect in a long time.

3) The important thing is to persist the strength training in a long-term.

4) The daily training a time, Weekly training a time, suspend training.

c) Relationship between stimulus frequency and strength growth. The overload training is an additive shock for the muscle of the body, and after the stimulation of weight, it can increase exercise intensity of nervous process, also can change the muscle structure and function, which would lead to the development of the muscle force.

If the strength training is set to $100 \%$ one day for a time, then to a two day strength training, the effect is $80 \%$, for a three day, the training effect is $60 \%$, for a 6 day, the effect is $40 \%$. This shows that the stimulation frequency and strength growth are closely related.

d) Static strength training. By using a fixed object to do resistance exercise, in this situation the muscle is in the form of isometric contraction force. This static strength training method is simple and the effect is very good. So, at present, it not only widely used in the sports industry, but also used in people's daily exercise.

When do Static strength training, it often gives the maximum muscle strength. A best practice time is 6-10 seconds, because it can effectively enhance the strength of purpose. In fact, you can also use 2-3 seconds repeatedly play to practice. A lonely person can do Static strength training because it doesn't need any equipment, which is a great feature to others.

\section{Endurance Strength Training}

a) Endurance. The breathing, circulation, blood characteristics, endocrine system, detoxification, excretion function, mental strength, muscular endurance and other aspects affect the improvement of endurance strength.

1) Respiratory function: The long distance running, wear a mask for high intensity exercise, and all with holding breathe training, such as diving, swimming are all suitable for strengthening the respiratory muscle training.

2) Circulatory function: In order to improve the function of the heart and blood vessels, it is need to take the intermittent training method and repetitive training method, which is better in the lasting training of overload. When choose a closely to the individual maximum overload training method to improve the endurance strength, the effect is not good, but choose the below $1 / 3$ individual maximum overload and repetitive training, the effect will be better.

3) Blood Traits: The number of Blood red ball has a special effect on endurance strength. The Mexico Olympic boards have proved that the effects are obviously better when training in the alpine hypoxia.

4) Detoxification, excretion function: Should be taken the nutrition (about intake water and salt for body) into Consideration

b) The training methods for improve endurance strength:

1) Continuous training method: when doing training, there is no not rest and continuous movement in it, such as long running, long distance swimming.

2) The intermittent training method: using $70-80 \%$ of a personal maximum overload amount to a maximum strength training and gradually shift to a hypoxia area for a repeated training. When take a break, it should be 
kept in a high efficiency state for the heart activity, which is aimed to increase the volume effect. In additional, Combine the high intensity exercise of anaerobic and aerobic interval training in the process of training has a good effect on improving the ability of muscle to bear oxygen.

For example, Practice running 150 meters for 5 times and each runs speed requirements 20-25 seconds; the pulse is improved to $170-180$ times per minute after each run. Then walking for $60-90$ seconds. If take a 90 seconds break and the pulse number are failed to recover to about 140 times, the training should be stopped.

3) Refresher training method: when taking the repetition training method, it should use more overloaded weight than the intermittent training method (full or $4 / 5$ maximum overload), and each exercise should be a complete rest and then repeat the training.

\section{Other Training Method}

a) Wild Running method: It is the transforms of the Continuous training method. For example, have a running across the forest or wild.

b) Mountain training method. Run with a certain speed in the terrain area.

c) Highland Training. Physical training in the highland hypoxia, in order to improve the ability of oxygen uptake.

d) Speed training.

All those factors of sensory organs, nervous system and muscle contraction speed determine the speed and agility degree.

The rules of the speed training:

1) Overload weight should be light.

2) Proceed in a high frequency.

3) Repeated training. Training the speed.

The methods of speed training are followed:

a) Sprint training method, with the fastest speed for a short distance run.

b) Continuous jump training method: overload $1 / 3$ of your own weight to have continuous jump training.

c) Moderate intensity overload training method: The purpose of training is not to increase the overload weight or frequency, but to shorten the time in completing certain frequency training.

d) The most effective methods to improve the response of athletes' speed are constantly changing the different signals, and the athletes can do corresponding action according to the signals. When in training the athletes should keep their muscle in a state of tension and ready to make any emergency responses.

All in all, when in the strength, endurance and speed these three qualities (including explosive strength and endurance strength training) physical training, the athletes should clearly the purpose of training in first, but also to consider the overload weight, repetition times, training methods and other requirements of the training. Have a Scientific arrangement before physical training will play a positive role to improve the quality of the body, which also make the effects of training more significant.

\section{Comprehensive Physical Strength Training}

To have an overall development of qualities for athletes, the athletes should take the circulating training method as a major training method.

The circulating training method:

1) Characteristics: it can compile the appropriate action that chosen into exercise programs. The purpose is to improve strength and respiratory function by repeating exercises. It has a good effect to deal with systemic forces, local forces, the body endurance, speed and explosiveness that depends on the selected exercise program.

2) It is different from weight training. Each exercise action is like myopia weight training method, but they have obvious differences.

Firstly, when you choose cycle exercise training method, you should mention that there is not a break between the action and the action. In the weight training method, it adds the endurance training of respiratory and circulatory systems.

Secondly, the load of the movement is smaller than the load of weight training method. Weight training method is based on the principle of two-thirds of the maximum load, but the Circuit Training Act is based on the amount 
of $1 / 2$ or less of personal training cycle maximum.

3) Prescription: for the timer of 30 seconds or one minute, measuring the maximum number of repetitions of each exercise, the half of it is the amount of an exercise. For example, an athlete can do push-ups 20 times in one minute. Then half of it, 10 times can be taken as the training prescription.

4) Choice of projects. In compiling the cycle exercises, the activities that all parts of the body muscles should get exercise are combined. The activities of the head, upper, front and lower limbs, hands and feet and other body parts should be taken into consideration for the exercise purpose. Due to the different aims of the exercise, focal points of the cycle action can be different.

Exercise actions can be increased or decreased on the basis of level of individuals and conditions and practice time. Each individual exercises according to the amount of reps to do it again for a loop (a group of) work to do three cycles (three groups). Time is usually about 10-30 minutes.

The selected training, everyone should measure their maximum number of repetitions times within a certain time, and then training themselves by its $1 / 2$ of highest exercise repetitions times, that is formulate the special prescription for each person's training.

For example A: $30 * 1 / 2=15$ times B: $20 * 1 / 2=10$ times C: $16 * 1 / 2=8$ times

Finally, I want to mention the nervous system and the jumping ability of the relationship. We already know that all those qualities would be produces the jumping ability in an instant on the ground under comprehensive of the speed, strength, coordination, flexibility strengths, however, what make those qualities outbreak of it in an instant at the same time? It is the motivation and motor systems that make this situation happened. That is to say, if you really want to be a top list person, you have to make your own motor system send the strongest muscles impulse signals by any possible methods. These intensive stimulations would force the muscle mass contraction fiercely and would emerge tremendous energy, and the fierce muscle mass contraction in turn prompt the movement and sensitivity of the nervous system, it will stir up more intense impulse for the body. Those two parts promote each other, so you can jump higher and higher.

\section{References}

Chen, X. R. (2005). Campaign training methods in transplantation. Beijing University of Physical Education.

Chen, Y., \& Liu, J. G. (2008). Development of physical strength training for track athletes. 12th National Sports Biomechanics Academic Exchange Conference Papers Series.

Guo, J. X. et al. (1991). Sports training. Beijing People's Sports Publishing press.

He, C. B. (2009). A Study on female Junior Sprinters Strength Training, 10.

Yu, Z. N. (1999). Track Events and Physical Training, 10, 6-9.

Zhou, Z. X., \& Zhang, F. (n. d.). Research on the arrangement features of high-level athletes in the strength training cycle. 7th National Conference of Sports Science Abstracts.

\section{Copyrights}

Copyright for this article is retained by the author(s), with first publication rights granted to the journal.

This is an open-access article distributed under the terms and conditions of the Creative Commons Attribution license (http://creativecommons.org/licenses/by/3.0/). 